\title{
Ir detrás del pez: estrategias de integración y difusión étnico-cultural en poblados pesqueros del litoral sur de RS-Brasil
}

\section{Go behind the fish: integration strategies and ethnic-cultural diffusion in fishing villages on seacost south of RS-Brazil}

\author{
Gianpaolo Adomilli ${ }^{1}$ http://orcid.org/0000-0001-8370-2267 \\ ${ }^{1}$ Universidade Federal do Rio Grande do Sul, Rio Grande do Sul, BRASIL. \\ Email: giansatolep@gmail.com
}

\begin{abstract}
Resumen
Este artículo aborda procesos de territorialidad relacionados con la configuración étnicocultural de pueblos pesqueros del litoral sur de Río Grande del Sur, Brasil, a partir de flujos migratorios, en parte desencadenados por conflictos relativos a la expansión portuariaindustrial y agroindustrial sobre los territorios de pescadores y agricultores de la zona costera, así como al crecimiento del mercado de la pesca en general. Ante estas situaciones de cambio, fueron generándose estrategias de supervivencia de esos grupos, que llevaron a su vez a la integración y difusión cultural entre diferentes grupos étnicos, considerando sus prácticas de trabajo y su lógica de parentesco. Así, busco presentar procesos de flujos migratorios de grupos étnicos impulsados principalmente por conflictos territoriales y por la orientación nativa de "ir detrás del pez". Se configura un "territorio de los excluidos socialmente", donde el borde de la playa se convierte en lugar que ofrece alimento (pescado), a través de las relaciones de trabajo y de acogida de las comunidades locales, a los que vienen de afuera. Se trata, pues, de abordar la formación de la configuración étnica de esa región articulada a la dinamicidad del modo de vida, en cuanto práctica creativa y de resistencia cultural.
\end{abstract}

Palabras claves: Territorialidad, flujos migratorios, pescadores, difusión étnico-cultural.

\begin{abstract}
This article deals about territoriality processes related to the ethno-cultural configuration of fishing villages of the south coast of Rio Grande do Sul (RS), Brazil, through migratory flux, partly triggered by conflicts related to the port-industrial and agro industrial expansion on the fishing territories of the coastal zone, as well as the development of the fishing market. Faced with these situations of change, survival strategies of these groups were taking place, leading in turn to the integration and cultural diffusion among different ethnic groups, considering their work practices and their kinship logic. Thus, I seek to present processes of migratory flows of ethnic groups mainly by the way of territorial conflicts and by the native orientation of "going behind the fish". It also points to the configuration of a "territory of the socially excluded", where the beach offers possibility of surviving these groups, through working and relationships between local communities, to those who come from outside. Then, I treat to approaching the formation of the ethnic configuration of that region as a creative practice and of cultural resistance, in this way the question of the cultural heritage is articulated with the dynamism of the way of life.
\end{abstract}

Keywords: Territoriality, migratory flux, fishermen, ethnic-cultural diffusion.

Recibido: 7 agosto 2017. Aceptado: 27 marzo 2018 


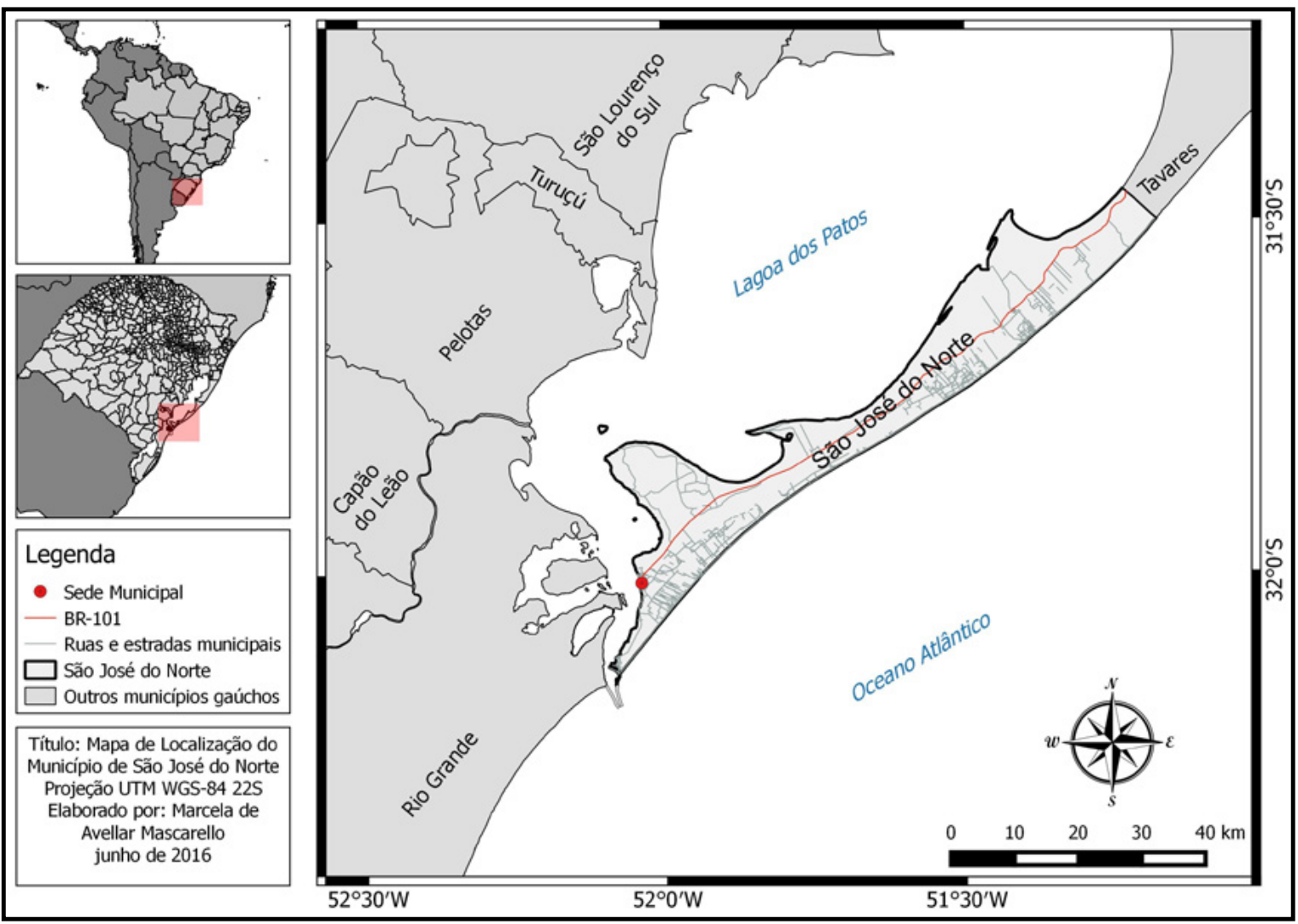

Figura 1. Mapa de localización de San José del Norte. Fuente: Santos, Puccinelli, Oliveira, Mascarello y Machado (2016).

\section{Introducción}

En este artículo, abordo algunos procesos de territorialidad y de configuración étnico-cultural de comunidades pesqueras del litoral sur del Río Grande del Sur (RS), principalmente en la región que abarca el estuario de la Laguna de los Patos y las playas del Atlántico (Figura 1). Para esto, retomo parte de la discusión y de datos levantados en mi investigación de doctorado, una etnografía realizada junto a pescadores del municipio de San José del Norte, ${ }^{1}$ la que busco extender en parte a los pueblos pesqueros vecinos, pensando en una configuración regional, así como sus relaciones con los pescadores de playas más lejanas, de Florianópolis y litoral sur de Santa Catarina (SC) (Figura 2).

En principio, se trata de lo que podríamos configurar como territorio de pescadores "azorianos" que,

1 Investigación (Adomilli, 2007) realizada entre 2004 y 2007. a lo largo de la costa, consiste en una extensión de playas, desde el litoral de Santa Catarina hasta el extremo sur del litoral de Río Grande del Sur, según lo señalado por Diegues (2000). Por otra parte, la noción de azoriano como grupo étnico de origen debe ser relativizada, una vez que son grupos mestizos, destacándose allí una significativa presencia de afrodescendientes, principalmente en la región que involucra a Río Grande y San José del Norte. Por lo tanto, trato de deconstruir la idea de un tipo folclórico y de ascendencia portuguesa, la cual reforzaría la apreciación de una supuesta identidad europea a expensas de otros grupos, como una forma de invisibilizar la presencia africana e indígena en la configuración étnico-cultural de los pescadores de la región. Por lo tanto, la noción de azoriano aquí presentada se refiere a un proceso de mestizaje, a partir de alianzas y de una operación de difusión étnico-cultural, principalmente entre grupos excluidos socialmente que, a su vez, se reterritorializaron en la actividad pesquera. 
De acuerdo con Diegues (2000) basado en Cascaes (1989), la costa de Santa Catarina y Río Grande del Sur está ocupada por los descendientes de las comunidades inmigrantes de las Azores, Madeira y Portugal continental, que se establecieron en el siglo XVIII y pasaron por siglos de miscigenación con indios y negros. En este sentido, también es claro que la costa de Santa Catarina y Río Grande del Sur fue habitada por diversos grupos indígenas, en su mayoría por los guaraníes, que practicaban la caza y la pesca, cuando el gobierno portugués trató de poblar la región con las familias de las Azores. También conviene mencionar que, específicamente en Río Grande y San José del Norte, hubo un gran flujo de esclavos negros por la vía del puerto de Río Grande, quienes posteriormente serían destinados al trabajo de las charqueadas.

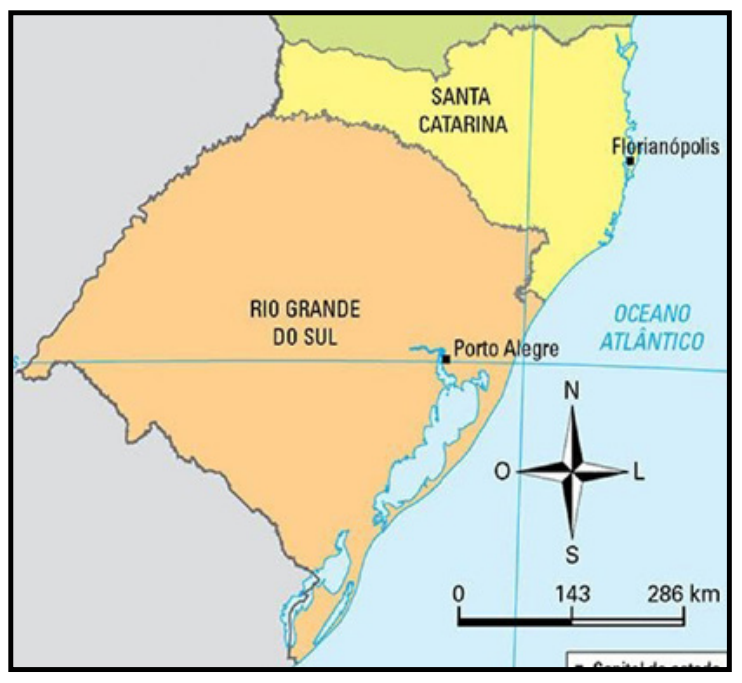

Figura 2. Mapa de los departamentos de SC y RS. Fuente: Adaptado de Editora do Brasil (2019).

Así, el contacto con remanentes de indígenas supone un proceso de readaptación de las técnicas de captura en la pesca local y de la absorción del conocimiento nativo sobre el ambiente biofísico de la región por parte de los colonos azorianos. Antes de la llegada de los colonizadores, poblaciones indígenas practicaban la caza y el extractivismo en la región, destacándose la pesca en el estuario de la Laguna de los Patos (Ihering, 2003; Kern, 1994). La violencia colonial y, en especial, las incursiones militares, así como las guerras en la región, desencadenaron un proceso de ocupación en el que parte de esas poblaciones fue diezmada y desplazada de una porción de sus territorios. Una fracción fue incorporada por los colonizadores, principalmente como mano de obra en el trabajo de salazón de las charqueadas (Niederle y Grisa, 2006).

En cuanto a la población negra, los descendientes de los esclavos permanecieron en las tierras de sus antiguos patrones, convirtiéndose en agricultores (Bunse, 1982). Fue recién a finales de la década de 1970, con la crisis de la cebolla y el proceso de expansión de la pesca local, que esa población negra rural comenzó a insertarse en la actividad pesquera.

Para Diegues (2000), las comunidades de descendientes de azorianos serían caracterizadas por prácticas tradicionales de pesca y agricultura. Por lo tanto la zona costera que atraviesa los dos estados se caracterizaba por ser un territorio que presenta ciertas configuraciones étnicas y culturales signadas por esas actividades. En este sentido, los autores definen azorianos como "población tradicional no indígena", detentores de saberes tradicionales, en la relación con el ambiente costero mediado por sus prácticas de trabajo y destacando inicialmente las labores agrícolas, teniendo la pesca como actividad complementaria. Según estos autores, los colonos azorianos ya practicaban la pesca en sus lugares de origen, siendo en el litoral sur de Brasil donde pasaron a combinar las actividades de agricultura y pesca, obedeciendo a un calendario complejo que articulaba esas actividades:

Los azorianos comenzaban a pescar la liza (mugil platanus) entre mayo y agosto, cuando abandonaban las actividades agrícolas. Como gran parte de ellos vivía aislados, en un comienzo garantizaban su subsistencia tomando prestadas técnicas y especies cultivadas por los indígenas, como, por ejemplo, la mandioca; el aceite para iluminación era retirado de peces y ballenas. Asimismo, las iglesias eran el punto de encuentro y donde iban los domingos, usando carros de buey. En el siglo XVII se dedicaban a la captura de la ballena con una embarcación llamada ballenera, la cual se ha vuelto fundamental 
para otros tipos de pesca practicados hasta el día de hoy (Cascaes, 1989, citado en Diegues, 2000, p. 53).

Dichas prácticas se irán modificando en un proceso de especialización de la pesca, sobre todo a partir de la mitad del siglo XX, caracterizándose por el pasaje de esos grupos dedicados a la agricultura hacia la pesca.

Por lo tanto, una primera cuestión que busco desarrollar será en torno al modo en que ocurrió la formación étnico-cultural de los poblados pesqueros del litoral sur del Río Grande del Sur y de qué forma se relaciona con la actividad pesquera. ¿En qué sentido se puede considerar a los pescadores locales como azorianos y toda la franja costera que envuelve el litoral sur de Santa Catarina y el litoral de RS como territorio de pescadores azorianos?

En este contexto, los procesos de territorialidad ocurren a través de flujos migratorios, los cuales están vinculados a las transformaciones de la actividad pesquera y también al paso de la agricultura hacia la pesca. Parte de la dinámica de transformaciones de la actividad pesquera se produjo en contextos de conflictos desencadenados por la expansión urbana portuario-industrial y agroindustrial sobre los territorios de agricultores y pescadores en la región costera, a partir de la cual se produjeron estrategias de integración y difusión cultural entre diferentes grupos étnicos considerando sus prácticas de trabajo y su parentesco.

A partir de estos aspectos, una segunda cuestión sería pensar cómo, en un tiempo reciente, hubo un proceso de ampliación de la actividad pesquera local, en el que, en pocas generaciones, se presenta un movimiento de la agricultura hacia la pesca.

Para ello, busco reflexionar, a partir de datos históricos y etnográficos, sobre el proceso de mutación y de continuidades y discontinuidades culturales relativas a esas comunidades pesqueras. Se trata, pues, de abordar la formación de la configuración étnica de esa región como práctica creativa y de resistencia cultural. De esta forma se articula la cuestión de la herencia cultural con la dinamicidad del modo de vida.

\section{Azorianos, portugueses, catarinenses $y$ afrodescendientes: la movilidad y la territorialidad en la pesca}

Podemos considerar que, en el litoral sur de RS, en el entorno de la Laguna de los Patos y en las playas del Atlántico es predominante una formación étnico-cultural de azorianos y afrodescendientes. Pero más específicamente en Río Grande y San José del Norte, se suma a estos un número significativo de inmigrantes portugueses y sus descendientes. En San José del Norte también se encuentran algunos pescadores portugueses que se concentran en el núcleo urbano de la ciudad; la mayoría de ellos pertenecientes a la última generación venida de Povoa de Varzim (Figuras 3 y 4), quienes se asentaron en la región -en un flujo migratorio que duró entre finales del siglo XIX y mediados del XX. H. Bunse (1982), un filólogo alemán que viajó por buena parte del Río Grande del Sur, realizó una descripción de los aspectos lingüísticos y etnográficos de la región, y describe San José del Norte en 1959, cuando estos portugueses se dedicaban a la pesca reunidos en los muelles y en las playas circundantes, utilizando barcos a remo o vela.

Al llegar a Brasil, estos pescadores se concentran en Río de Janeiro, donde se organizan en comunidades de trabajo en torno a la pesca, aunque posteriormente una parte de ellos se desplazó a otras regiones del país, principalmente hacia las ciudades de Río Grande y San José del Norte. Estos pescadores actuaban en el estuario de la Laguna de los Patos y en la costa del océano Atlántico a través del sistema de parelhas - barcos movidos a remo y equipados con redes que operaban con un gran contingente de tripulantes.

Tuve la oportunidad de entrevistar a uno de estos inmigrantes portugueses en San José del Norte, quien me contó los detalles de su venida a Brasil en 1928. En primer lugar, estuvo en Río de Janeiro, donde pasó una temporada antes de establecerse permanentemente en San José del Norte. En su casa realizamos una entrevista en la que relató su trayectoria ligada al flujo de pescadores de Povoa de Varzim hacia esa región: 
Esto aqui eran todos portugueses que trabajaban, era todos pescadores. Tenia más de quinientos o seiscientos portugueses, todos de Povoa de Varzim. Nuestro continente era de Póvoa de Varzim, distrito de Porto. Cuando vine aqui, ya habia andado en el mar. Vine desde Portugal hasta aqui navegando [...]. Comencé cuando era niño. Trabajaba alli. En ese momento me casé, fui maestro, y tuve la posibilidad de tener una parelha y entonces... Compré para trabajar y fui trabajando y pagando la pareja. Me quedé dueño de la pareja. Y tenía de todo, galpones y pareja... Y tanto en la laguna como en el océano era la misma pareja. Estaba compuesta por cinco o seis embarcaciones. Tenia redes, doscientas redes... Se llamaba pareja porque tenía redes para todo y estaba compuesta por cinco o seis embarcaciones. Todas iguales, y cada una con un maestro. Y eran todos de la comunidad (Zé André, com. pers., 2004).

El cambio a Brasil mejoró su condición de vida, sobre todo en relación a la pesca, si se compara con las dificultades vividas por portugueses en el ejercicio de la actividad en su tierra natal.

Aqui ganaba mejor. Porque alli, daba la pesquería, daba. No se podía decir que no daba. Pero era solo en el océano. Y existían las invernadas. ;Alli, en el tiempo del invierno... era muy fuerte! Entonces, en aquellos cuatro o cinco meses, nadie trabajaba. Que no se podia trabajar. Eran temporales, era mar alto... Tenían los obstáculos de la vida, del trabajo. Entonces tenian los pescadores que se iban para allá. Que aqui no tenía esas invernadas. Era de la barra adentro. Y en Río de Janeiro, también se trabajaba. Tenia mucho pescador alli en aquella época. Y el mar allá en Río de Janeiro es más tranquilo. Aqui es muy bravo. ¿Y alli, en Portugal también era muy bravo! $Y$ con las lanchas de aquel tiempo... lanchas, lanchones de boca abierta... ;Sufrian mucho! Ahora... también la pesca alli ahora es moderna. Tiene barcos a vapor y todo. Entonces, cuando quiere venir es solo encender el motor. En aquel tiempo no. ¡Era paño! ¡Y remo!
Se llevaba una noche... o un dia para llegar a tierra. Entonces, la vida se tornaba muy dificil. $Y$ aqui tenía más facilidad, porque era mar y mar [océano y laguna]. Daba mucho pescado. iNo daba dinero, pero daba mucho pescado! (Zé André, com. pers., 2004).

Portadores de una gran experiencia de pesca, estos pescadores introdujeron nuevas técnicas de captura e iniciaron un proceso productivo comercial en la región, pasando a organizar una pequeńa producción destinada al puerto de Río Grande. El pescado se salaba, inicialmente para consumo propio, pero posteriormente algunos de ellos adquirieron capital suficiente para crear empresas de pescado salado, que pasó a ser exportado a la región sudeste de Brasil en un proceso de consolidación de las industrias de conservas de pescado a través del suministro del mercado nacional. Las industrias de pescado enlatado y sus exportaciones a la región sudeste de Brasil, incluso en el siglo XIX, fueron observadas por Ihering (2003). De acuerdo con Martins (2002), además de los pescadores de Povoa de Varzim, el desarrollo de la actividad pesquera atrajo a otros inmigrantes portugueses que presentaban prácticas de producción mercantil, que más tarde se convierten en el negocio de la pesca, generando cambios que condujeron a la formación de un parque industrial de la pesca en Río Grande.

Además de la pesca para la comercialización, el sistema de parelhas implicó una división del trabajo, en la cual se destaca la figura del "patrón", quien es responsable de todas las decisiones en las pesquerías y en algunos casos dueño de la embarcación, ${ }^{2}$ y los demás pescadores que componían la tripulación bajo el mando de aquel. Con el desarrollo de la actividad, algunos maestros dejaron de pescar y se convirtieron exclusivamente en dueños de una o más parelhas, en las que empleaban a las tripulaciones.

2 El patrón es responsable de las pesquerías, en el sentido de que comanda todo el trabajo en la embarcación y presenta, durante el viaje, total autonomía en sus decisiones. Por lo tanto, estas funciones eran preferentemente desempeńadas por alguien experimentado. La experiencia se funda en la capacidad de garantizar el éxito en la captura del pescado. 


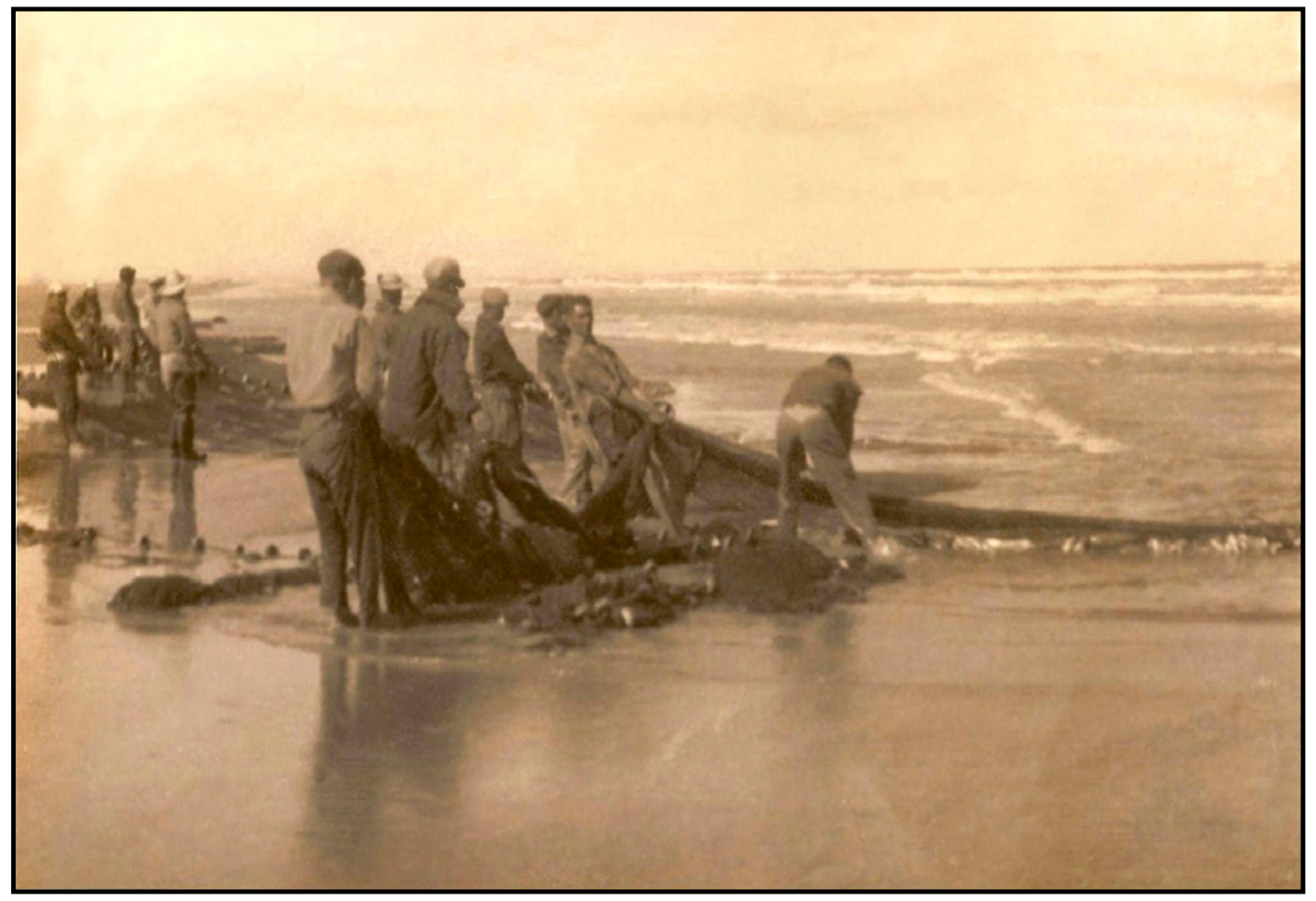

Figura 3. Pescadores portugueses en San José del Norte (s/d). Fuente: Imagen cedida (Adomilli, 2007).

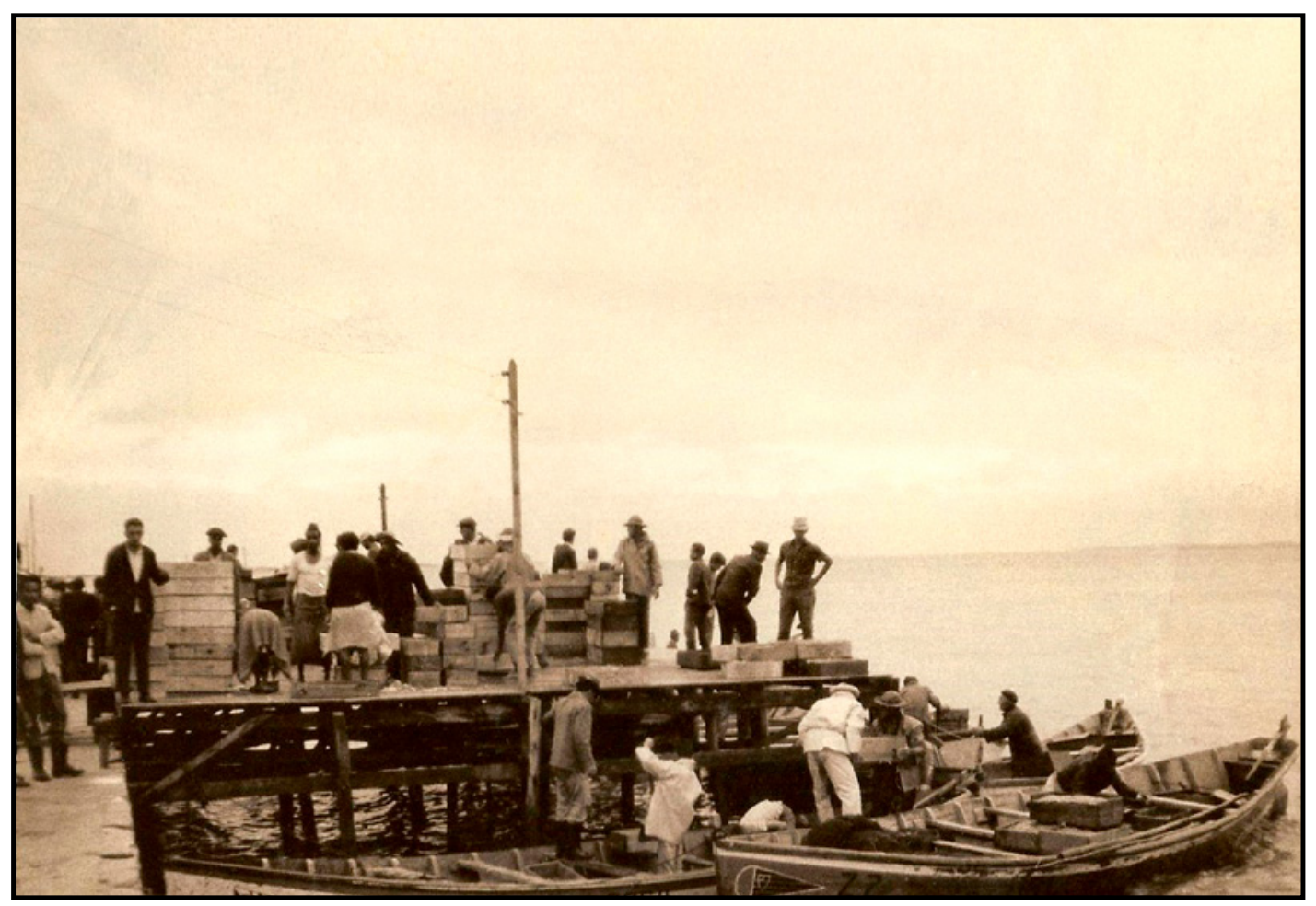

Figura 4. Pescadores portugueses en San José del Norte (s/d). Fuente: Imagen cedida (Adomilli, 2007). 
Por lo tanto, estos pescadores introdujeron una dinámica de pesca mercantil en la región en donde hasta entonces se practicaba una pesca de subsistencia, la mayoría de las veces en complementariedad con la agricultura.

Conforme Orlando (1989), los portugueses disponían de técnicas de pesca que les conferían una mayor capacidad de captura en relación con los descendientes de azorianos, que se dedicaban de preferencia a la agricultura y pocos eran exclusivamente pescadores. Los pescadores nativos, a su vez, difícilmente se sometieron a trabajar para los portugueses, ya que eran pescadores-agricultores, con un ritmo pesquero diferenciado, que no se basa en la producción de mercancías, sino en el trabajo como unidad familiar, centrado en la subsistencia. Por este motivo, inicialmente la relación entre los pescadores portugueses y los nativos, descendientes de los azorianos, fue de poco contacto. Además de la mayor tecnología y experiencia pesquera, en la perspectiva de una pesca profesional en relación a los azorianos, los pescadores de Povoa do Varzim formaron una comunidad de trabajo étnico, predominando los matrimonios entre ellos como forma de mantener sus tradiciones, constituyendo comunidadades con mucha cohesión y relativamente cerrada.

A partir de la década del cuarenta, esta situación comenzó a ser alterada debido a la coyuntura de desarrollo de la actividad pesquera en la región, con mayor penetración del mercado y de la industria, asociada al fin de la migración de esos pescadores hacia Brasil.

En este contexto, pescadores catarinenses se desplazaron desde la costa de Santa Catarina hacia esta región, detrás de las cosechas de pescado provenientes del sur. Las dificultades para seguir con sus parelhas llevaron a los portugueses a emplear principalmente pescadores de Santa Catarina, que aparecían en grupos cada vez más grandes. El problema de la escasez de personal y la relación con los catarinenses finalmente desestructuraron las parelhas portuguesas, que fueron siendo sustituidas por las parelhas catarinenses, de mayor número y conformadas por menos hombres (Orlando, 1989).
En ese sentido, Rial (1988) observó que los catarinenses permanecían durante largas temporadas en el sur, trabajando en las pesquerías y a veces retornaban a Florianópolis casados con mujeres nativas del litoral de Río Grande del Sur. En mi investigación, analizando las trayectorias de los pescadores de San José del Norte, observé que también ocurría que muchos de esos catarinenses se establecían en la región gracias a estos matrimonios. De esta forma, el parentesco entre los pescadores (y entre pescadores y agricultores) acabó extendiéndose a lo largo del territorio costero entre los dos departamentos, teniendo como referencias principales las playas de Santa Catarina, sobre todo Florianópolis, y de Rio Grande del Sur, principalmente San José del Norte y Río Grande. La naturaleza de esos matrimonios remite a las alianzas y a la receptividad de las comunidades de pescadores-agricultores de origen azoriano, como grupo abierto a los que vienen de afuera.

A través de los desplazamientos a lo largo de la costa de Río Grande del Sur, muchos de esos pescadores acabaron estableciéndose en la región en virtud de matrimonios con mujeres nativas, como ya señalamos, que favorecieron una mayor integración a la población local, formada principalmente por agricultores y pescadores agricultores. Estos se dedicaron a la pesca por la interacción con los portugueses y personas de Santa Catarina, debido principalmente al proceso de vaciamiento humano del campo y su consecuente concentración en los centros urbanos.

En la costa de Santa Catarina, la expansión urbana, especialmente de Florianópolis, impulsada por la especulación inmobiliaria derivada del turismo, generó al desplazamiento de una serie de comunidades de pescadores agricultores de origen azoriano, que pasaron a dedicarse en forma exclusiva a la pesca.

Las playas, que eran ocupadas únicamente por pescadores, despertaron el interés de la especulación inmobiliaria impulsada por el turismo y por el aumento poblacional de Florianópolis, en el contexto de un proceso de "gentrificación" del litoral. Así, se observa la utilización del borde costero para actividades ligadas al turismo en detrimento de la pesca, como, por ejemplo, la construcción de resorts y otros 
emprendimientos habitacionales en playas habitadas por comunidades pesqueras.

La intensidad de este proceso acabó por desorganizar el modo de vida de esas comunidades -que se basaba en prácticas de trabajo tradicionales-, con ciertas reglas de ocupación de espacios de pesca en la franja costera. El exceso de pescadores generó conflictos y disputas sobre dichos espacios. Las innovaciones tecnológicas y la expansión del mercado de la pesca en Santa Catarina también llevaron a una intensificación de las capturas provocando una "sobrepesca", fenómeno mundial que se refiere al aumento progresivo de esfuerzo de captura con miras a mantener un padrón productivo alto pero incompatible con la renovación de los stocks pesqueros.

Como estrategia de supervivencia, buena parte de esos pescadores se fue mudando de playa en playa, en movimientos estacionales para Rio Grande do Sul, São Paulo y Río de Janeiro (Diegues, 1983). En sus estudios con pescadores de la Lagoa da Conceição - SC, Rial (1988) descubrió que, además de la territorialidad marítima local, esos pescadores también presentaron una relación territorial con los mares de otras regiones. Basado en sus testimonios, Rial cuenta sobre los viajes al mítico mar de Rio Grande, cuyo medio de transporte hasta la década de 1940 era a vapor y luego, con la construcción de las carreteras, fue sustituido por camiones. Estos viajaban por la actual rodovía BR-101 hasta la costa al norte del RS y luego seguían al sur varios kilómetros por la arena, recorriendo la frontera de playa hasta San José del Norte (Rial, 1988).

Paralelamente, en el litoral sur de Río Grande del Sur, desde el siglo XVIII, la contribución de los azorianos al trabajo agrícola en la región reveló una producción diversificada, con énfasis en la comercialización de trigo, hasta principios del siglo XIX. Con la disminución de la producción de trigo, el cultivo de cebollas, que presentó buenos resultados en el suelo arenoso, pasó a ocupar gradualmente la escena agrícola local.

La fase de modernización de la agricultura en Brasil, iniciada a finales de la década de 1960, caracterizada por el uso de máquinaria y pesticidas, incentivó las plantaciones de cebolla, una actividad que era tradicional, pero ahora a gran escala. Así, se pasó al monocultivo, en detrimento de la agricultura familiar, más diversificada, resultando en concentración agraria, que fue decisiva para el fenómeno de éxodo rural.

El resultado frente a la escasez en el campo fue el desplazamiento de familias enteras al núcleo urbano de San José del Norte y, de esta forma, del paso del trabajo en la agricultura a la pesca. Este proceso se asemeja a lo que ocurrió en el litoral de Santa Catarina. Sin embargo, en vez de la expansión urbana, aquí el factor determinante parece haber sido el modelo de desarrollo agrícola, más específicamente el monocultivo de la cebolla y, en las regiones vecinas, el monocultivo de arroz en el modelo pecuario generó concentración de tierra en pocas manos.

Sin embargo, además del impacto de la modernización de la producción agrícola, la cuestión del abandono del campo no puede ignorar el problema de la falta de tierra en el marco del creciente aumento de la población rural. La cuestión de la herencia de la tierra en las familias nucleares campesinas fue abordada ampliamente en estudios socioantropológicos sobre campesinos, destacándose los estudios clásicos de Bourdieu (1958), Wolf (1976), Queiroz (1972) y Woortmann (1995, 1997). Se trata de un tema que presenta una cuestión más fundamental, que refiere al problema ya discutido en estudios clásicos que consideran el problema de la "falta de tierra" para el aumento de la población campesina. Para Eric Wolf (1957), el proceso de herencia puede ser "para compartir la tierra" o "sin compartir la tierra", sin embargo, siempre implica la migración de campesinos.

Junto con los descendientes de azorianos, el movimiento de la agricultura hacia la pesca absorbió comunidades negras rurales que se establecieron en la región durante el período esclavista y que vivían hacía siglos aislados y explotados para el trabajo en el campo. Buena parte de las narrativas de los pescadores acerca del tiempo de abundancia vinculada a la pesca es precedida por trayectorias familiares de dificultades económicas, en un contexto en el que el litoral y la actividad pesquera se presentan como el lugar de los excluidos socialmente. Para las familias que abandonaron el campo, la inseguridad 
de una larga espera de los resultados de la cosecha es sustituida por el retorno inmediato de las pesquerías; no exactamente de dinero, pero sí de pescado, garantizando así el alimento.

Esta situación puede ser observada a partir de la trayectoria de Claudio, un afrodescendiente, patrón de un barco, a quien entrevisté y que relata haber venido desde el interior de San José del Norte hacia la orilla de la playa con su familia, cuando era niño, huyendo del hambre:

Entonces la gente vino a parar aqui. [....]. En aquella época se usaba corriente en los neumáticos, para pasar por esos bañados. No tenía acceso al interior, era por la playa. Si no tuviera playa, no tenía cómo llegar alli [...]. Entonces la gente llegó aqui con mucha dificultad. ;Mucha dificultad! [...]... Y la abundancia era tanta de pescado que, en una época de esas de verano, comía el pescado que quería [...]. Y yo venía de alli a recoger, a buscar lo que comer. No teniamos qué comer; entonces yo llevaba una harina en la casa de uno, otra cosa en la acasa de otro, y yo venía por la mañana a buscar el pececito aqui (Claudio, com. pers., 2004).

Así, el litoral se presenta como un espacio de refugio de los socialmente marginados, sumando a los excluidos del campo a aquellos pescadores sin la posesión de los instrumentos de trabajo y de acceso a un territorio de pesca. La playa, espacio de uso común y al mismo tiempo liminar entre la tierra y el mar, consiste en un espacio límite, en el que se mezcla el aislamiento espacial y el nomadismo de los excluidos socialmente, donde ingresan en la pesca a través de su trabajo en las tripulaciones.

Para Cordell (2001), que trata de la organización social y de los "bordes" de Bahía (BA), el proceso de marginación social, especialmente en relación con la posesión de la tierra, hizo que las áreas de manglares y lagunas rasas se convirtieran en "punto de no retorno-refugio”. Cordell también observó que las comunidades pesqueras fueron predominantemente conformadas por poblaciones negras excluidas de las plantaciones del interior, presentándose un proceso similar al de San José del Norte.
De esta forma, los hombres se empleaban en la pesca, ya que en el sistema de parelhas surge el dueño de la parelha, el patrón y la tripulación en general. Estos últimos son pescadores sin posesión de los instrumentos de trabajo. Por su parte, las mujeres eran empleadas en las industrias de conservas de pescado salado, proporcionando mano de obra para el parque industrial pesquero que se formó en la región. En el borde de las playas del canal, antes áreas bastante amplias, consideradas fuera de la ciudad, se formaron pequeñas villas de pescadores, así como en la costa del océano en lugares donde se crea un vínculo con las personas oriundas del campo, que aparecen para ayudar en las pesquerías a cambio de pescado. El proceso de abandono de la agricultura hacia la pesca se asemeja a lo ocurrido en el litoral de Santa Catarina. Sin embargo, en lugar de la expansión urbana, aquí el elemento determinante parece haber sido el modelo de desarrollo agrícola, sea en el monocultivo de la cebolla y del arroz o incluso en las estancias con vocación ganadera, llevando a la cuestión de la concentración de tierras. Pero además del impacto de la modernización de la agricultura, la cuestión del abandono del campo no puede descuidar el problema de la falta de tierras para el creciente aumento de la población rural.

Se puede afirmar que la pesca artesanal en el Río Grande del Sur comienza con estos pescadores portugueses, a la que se sumarán los pescadores de Santa Catarina. El proceso de vinculación de la pesca artesanal con el mercado es narrado por los pescadores como referencia de una "época de abundancia” de la pesca, cuya producción de camarón y pescado era increíblemente superior a la capacidad de consumo local. Ni las industrias de pescado salado -que trabajaban toda la noche- ni tampoco la distribución de gran cantidad de peces y camarones entre las personas de la comunidad impedían que toneladas fueran desperdiciadas (o utilizadas como abono, principalmente para las plantaciones de cebollas), ya que no había tecnología para la conservación del pescado. Asimismo, el proceso de rápida descomposición del pescado hacía que los pescadores vendieran su producción a valores irrisorios para la industria de pescado. 


\section{Ir detrás del pez a las playas de la laguna y del océano: un camino de pocas generaciones}

Históricamente, el municipio de San José del Norte se desarrolló en relación con Rio Grande por medio de las actividades portuarias, combinadas con la pesca y el cultivo de cebollas. Entre los habitantes, se observa la referencia a una división entre "aquellos que cruzan y aquellos que no cruzan las aguas" (Adomilli, 2007), lo que implica una relación de este territorio con la laguna y el océano, de un relativo aislamiento geográfico, como de insularidad, donde cruzar o no las aguas significa simbólicamente vivir en un territorio anfibio.

En este sentido, la relación con el ambiente acuático se refiere a las prácticas de trabajo y a los ritmos de la pesca, que involucran a las familias en sus lazos de parentesco y solidaridad. Por otra parte, este aislamiento es relativo, ya que las aguas surgen como nuevas rutas de comunicación entre pescadores de diversas localidades. Además de la configuración territorial costera de pescadores azorianos, el mar y las lagunas también revelan otras rutas: la de la comunicación entre pescadores del entorno de la Laguna de los Patos y también de los viajes más largos, en las pesquerías en barcos que trabajan en el Atlántico, mar adentro.

Un aspecto de esta movilidad y comunicación es el sentido otorgado al desafío de las capturas de peces, es decir, las pesquerías y la relación con el mercado, impone a los pescadores ir detrás del pez. En cierta forma, los flujos migratorios y la difusión étnica entre portugueses, catarinenses y nativos de la región se dieron en emprendimientos pesqueros que adquirieron ese sentido. Así, los grupos pesqueros tratan de buscar nuevos sitios de pesca, más allá de sus territorios originales Lo mismo ocurrió en la comunicación entre comunidades pesqueras del entorno de la Laguna de los Patos, en especial en el caso de la Isla de la Feitoria, a partir de la lógica y demanda de las capturas de pescado.

En las narrativas sobre el tiempo en que se pescaba en la Laguna de los Patos, la Isla de la Feitoria surge como una referencia importante. En el estuario, cerca del municipio de Pelotas, este local albergaba a los pescadores de los municipios alrededor de la laguna, principalmente entre las décadas del setenta y ochenta. Pescadores de Pelotas, de Río Grande, San José del Norte y de San Lorenzo del Sur acampaban en la isla con sus familias, permaneciendo durante los períodos de pesca, que duraban algunos meses, sobre todo en la cosecha del camarón, desarrollada durante el verano.

Actualmente esta isla está prácticamente abandonada, debido a una disminución considerable de las especies que eran capturadas, lo que alejó a las familias de pescadores. Incluso los habitantes de la isla optaron por mudarse a la Colonia Z3, el poblado pesquero más cercano, que se convirtió en un barrio de la ciudad de Pelotas.

Las pesquerías en la Isla de Feitoria se refieren a una coyuntura de consolidación de la figura del intermediario, que compraba la producción de los pescadores. Durante este período, surgieron nuevas técnicas de pesca e innovaciones tecnológicas que fueron fundamentales para los cambios en el sector pesquero, como el hielo, que posibilitó el almacenamiento en mayor cantidad, y el motor, que pasa a ser utilizado en las embarcaciones para sustituir al remo y las velas, dando lugar a los barcos motorizados.

Con botes y canoas a motor (Figura 5), los viajes más distantes como los realizados hasta la Feitoria facilitaron el encuentro y la comunicación entre pescadores de diferentes localidades. Por otra parte, la imagen del pescado levantado por la horca, herramienta utilizada por los agricultores, presente en narrativas de pescadores, remite a un tiempo de abundancia asociado al cambio de prácticas agrícolas para las pesquerías, en un período de pesca artesanal, en el que aún no era necesario cuidar de la integridad del pescado, de acuerdo con las demandas actuales del mercado de la pesca.

Aunque se identifica una división del trabajo como unidad familiar, en la que los hombres pescan y las mujeres, además de descascarar el camarón, generalmente participan de la pesca en botes y canoas, es importante aclarar que una proporción de ellas también laboraban en pequeñas embarcaciones, 


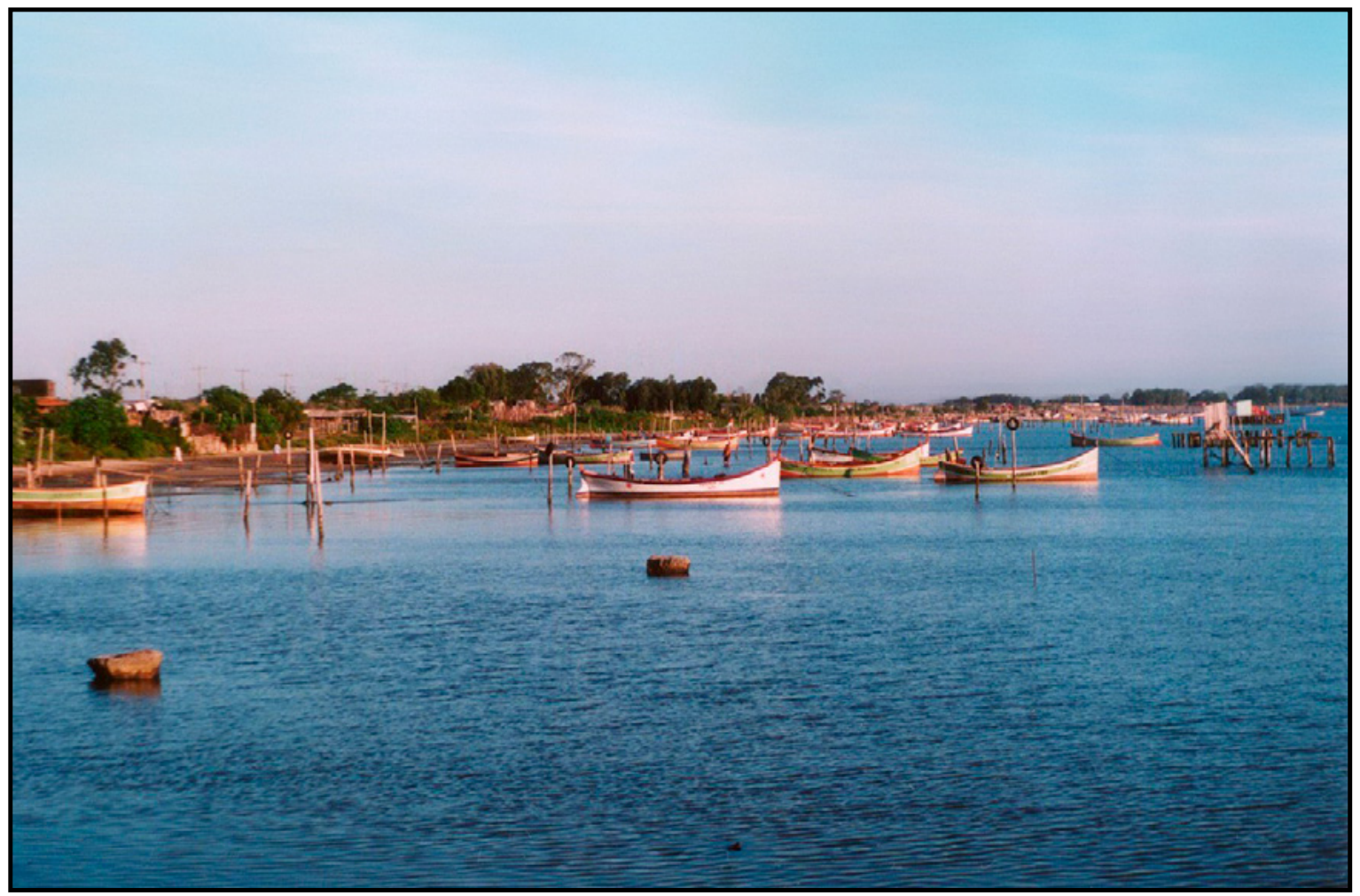

Figura 5. Playa de pescadores en la Laguna de los Patos - San José del Norte. Botes y canoas a motor. Fuente: Adomilli (2007).

solas, con el marido o los padres. En las familias de pescadores, las mujeres tradicionalmente trabajaban en la preparación del pescado seco, en el proceso de salar, así como en las actividades de pelar el camarón, sea para consumo propio, sea para la venta. En esos campamentos las familias enteras también realizaban un arrastre con redes en aguas poco profundas, en la pesca de camarón.

Ya habituadas al trabajo de limpiar (extraer las vísceras) y preparar el pescado, que era salado y secado, en el núcleo familiar, las mujeres también trabajaban dentro de las empresas de procesamiento de pescado. El trabajo en las industrias de pescado salado, operando noches enteras, no daba cuenta de toda la producción del pescado. Con las fábricas de congelados, el procesamiento del pescado se hizo más rápido, mientras que el número de trabajadores en estas fábricas disminuía. En la década del setenta, había dos subsidiarias de esas empresas en San José del Norte (Figura 6), con sede en Río Grande. La sustitución de los puntos de venta por las fábricas de congelación de pescado y el aumento del parque industrial del Río Grande, llevó a Confrío, una de esas fábricas, a instalar una subsidiaria en el sector del muelle de San José del Norte, absorbiendo mano de obra local.

En San José del Norte, la pesca embarcada comenzó recién en la década del ochenta, con el surgimiento de la flota de embarcaciones equipadas con redes de enmalle y con algunas tecnologías disponibles de barcos de pesca industrial, como sondas y brújulas (Figura 7). En este contexto, los pescadores locales dedicados a la pesca oceánica forman parte de una flota costera que presenta una transición de la pesca artesanal, basada principalmente en las relaciones familiares, en el conocimiento natural de los peces y el medio ambiente acuático en general, y baja tecnología, modelo con mayor implicación con el mercado. En cierto modo, se trata de una forma de rearticulación generada frente a cambios desencadenados por la actividad pesquera industrial, atribuyéndose su origen a las políticas gubernamentales 


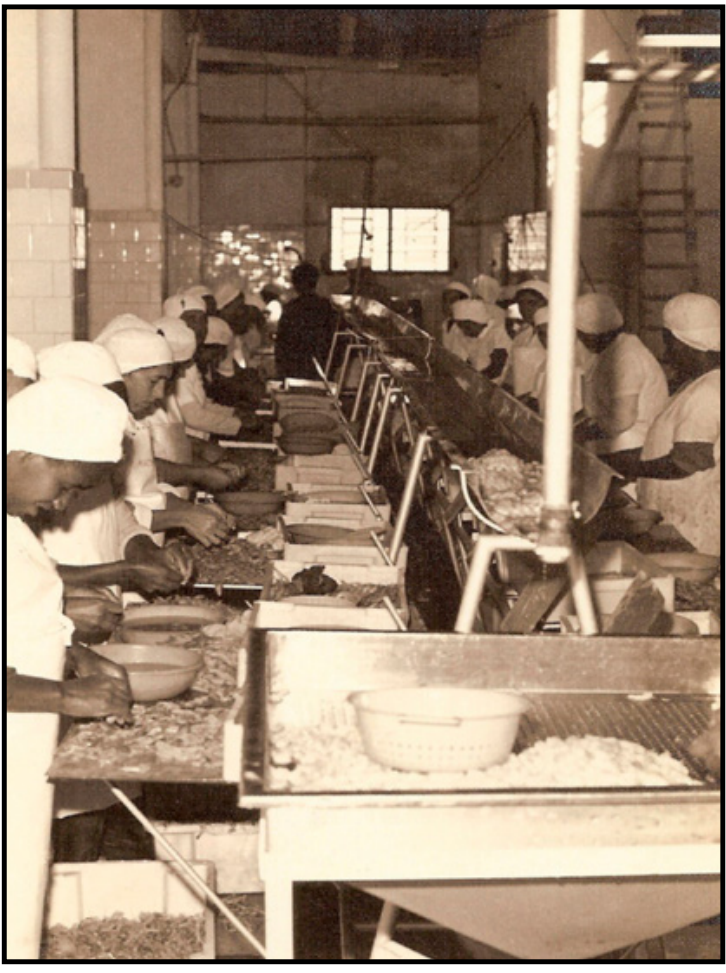

Figura 6. Trabajo con el camarón en una fábrica de pescado a fines de la década del setenta.

Fuente: Imagen cedida (Adomilli, 2007).

para este sector, realizadas a partir de la década del sesenta y que benefició a una diversidad de propietarios de parelhas de la Laguna de los Patos a través de incentivos fiscales, con vistas a un mayor equipamiento y adquisición de grandes embarcaciones. Estos, teniendo en cuenta la disminución de la pesca en la Laguna de los Patos y con mejores condiciones económicas, se convirtieron en armadores, invirtiendo en barcos, equipando redes y volviendo a la pesca mar adentro.

Por lo tanto, la mayoría de los propietarios de locales son descendientes de inmigrantes portugueses, mientras que las comunidades locales de pescadores, como ya hemos señalado, están conformadas por trabajadores agrícolas excluidos, junto con pescadores de Santa Catarina que fueron "detrás de los peces”, en búsqueda de nuevos espacios. La explotación de los recursos naturales que acompañó las inversiones de una industria del sector pesquero expandió la capacidad de captura, de forma que en las décadas siguientes se agravó aún más la escasez de pescado.
La pesca a gran escala en la zona costera marítima y la relativa escasez en la laguna, tras una intensa explotación de sus recursos naturales, hicieron que las empresas de pescado cerraran las puertas y la Confrío fuera trasladada a la región norte del país.

En este contexto, a finales de la década del ochenta, algunos armadores de barcos con redes de enmalle adquirieron barcos más grandes, pasando a pescar en aguas más profundas.

En los años noventa, se hizo evidente el agotamiento del modelo gubernamental de desarrollo para el sector pesquero implementado desde la década del sesenta, sobre todo como consecuencia de la acción de grandes barcos pesqueros, señalada a través de la decisión del índice de captura en las pesquerías y de las quiebras y fusiones de empresas, con una sensible reducción de la mano de obra local.

En términos globales, la problemática va más allá de la pesca local y de la política pesquera ejecutada por el Estado, pues se inserta en el ámbito de la captura mundial, destacándose la modernización de las flotas pesqueras que, con los adelantos tecnológicos empleados, tienden a pescar cada vez mayores volúmenes, llevando a la sombría perspectiva de un agotamiento de las capturas de pescado.

Los estudios acerca del impacto de las pesquerías en las poblaciones pesqueras llaman la atención sobre los altos porcentajes de descarte por captura de barcos de la pesca industrial. Esto significa que, además de los peces pequeños, varias especies capturadas no son aprovechadas y son arrojadas al mar ya sin vida. En el caso de los pescadores embarcados con red de enmalle, la práctica de pesca se caracteriza por la utilización de la red de espera, considerada una pesquería más selectiva que la practicada por los barcos de pesca industrial. Por otra parte, tal pesca ha ampliado considerablemente su extensión de redes y ha permanecido más tiempo en el mar, con el propósito de retomar la productividad del período de las primeras embarcaciones. El hecho de que la pesca con red de fondo inicialmente practicada durante el invierno se utilizara todo el año, incluso durante el verano, revela el contexto de la problemática ambiental que involucra directamente a esa pesquería. 


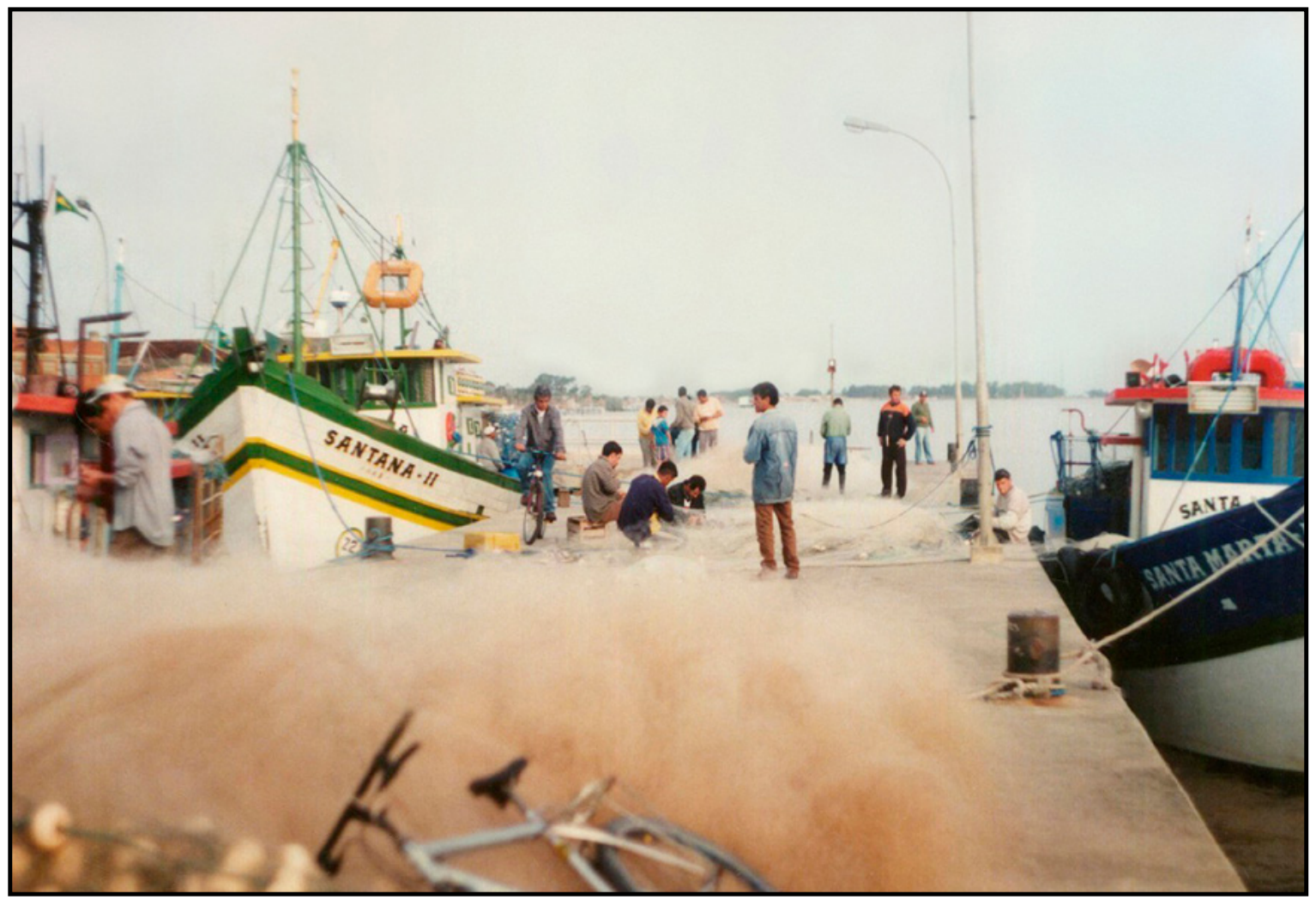

Figura 7. Puerto pesquero de San José del Norte, 2004. Fuente: Adomilli (2007).

Esta situación remite al hecho de que las problemáticas ambientales las instalan justamente las políticas ambientales, donde la vigencia de políticas de fiscalización con poder coercitivo y desigual entre los actores sociales involucrados incluye esta lógica en una perspectiva individualista, según revela Lima (1999, 2004). En ese sentido, Lima (2004) estudia el dilema de la administración de los conflictos en el espacio público brasileño, en el cual la tendencia a naturalizar la desigualdad a través del asentamiento legal de una igualdad formal, particularizando así los derechos humanos, se refiere a un sistema compuesto por reglas que están sujetas a la interpretación, llevando a la cuestión de la autoridad interpretativa y de la jerarquía.

Algunos pescadores de botes que se incorporaron a los barcos con redes de enmalle también han experimentado experiencias en grandes barcos de pesca industrial, en viajes a otras regiones del país, demostrando con ello una gran movilidad relacionada a la dinámica de intercambio existente en el ámbito pesquero.
Tanto en relación con los grupos que practican la pesca en botes y canoas a motor como en los de los barcos, se destaca el conocimiento acerca de las pesquerías. Este saber es transmitido oralmente de una generación a la otra, así como a través del acompañamiento en el trabajo, de acuerdo con las relaciones de parentesco y amistad que rigen esas actividades. Las nuevas generaciones incorporan nuevas experiencias en la dinámica que implica un movimiento de pocas generaciones de prácticas agrícolas hacia la pesca y de esta a lugares cada vez más distantes en el mar.

Se constata un cierto drama sobre este proceso, en el que la pesca embarcada, al consolidarse como alternativa a la pesca en la laguna, engendra una serie de cambios en los ritmos de vida. La dependencia en relación al mar se refiere, primero, a una temporalidad diferenciada. Esta situación demuestra de qué modo hay una configuración cultural que se construye de acuerdo con la transformación local de la actividad pesquera. El hecho de que la pesca 
se realiza en un punto cada vez más distante de la costa, gradualmente pasando de la tierra a las zonas costeras y lagunares, y de estas a la pesca en el océano, mar adentro, se experimenta con temor cuando se constata que el mar consume hombres, mas es también donde las nuevas generaciones agregan conocimientos a aquellos heredados a través de la transmisión generacional en el trabajo familiar. En ese sentido, estas transformaciones implican prácticas creativas a partir de las demandas de mercado de la pesca, mediadas por los aparatos tecnológicos.

\section{Consideraciones finales}

En este artículo, intenté demostrar cómo, en la franja costera del litoral sur de Rio Grande do Sul, especialmente en el municipio de Sáo José do Norte, se produjo un proceso de difusión cultural, a través de flujos migratorios, siguiendo la lógica de la pesca, en constante reelaboración de las demandas de la producción y de la industrialización. Las tensiones y reconversiones determinadas por los cambios sistemáticos derivados del proceso de industrialización en el sector pesquero se presentan en contextos de conflictos cuyas prácticas se refieren a los límites entre la condición de vulnerabilidad de esos grupos y sus estrategias de supervivencia. En este contexto, surgen algunas cuestiones relacionadas con los procesos de territorialidad y de nuevas prácticas y conocimientos, que se pueden ver aquí como un patrón de mutación. En este, condiciones adversas revelan al mismo tiempo un proceso de cambios en pocas generaciones, que remite a experiencias oriundas de prácticas agrícolas para pescar en las playas del Atlántico y de estas hacia el estuario de la Laguna de los Patos, y posteriormente en la pesca a mar abierto.

En este sentido, las estrategias de continuidad en el tiempo están ligadas a una unión sociocultural específica, que se encuentra en constante reelaboración, en una situación de territorialidad marítima ligada a las formas dinámicas de su modo de vida.

Los desplazamientos incesantes y ocupaciones se orientan hacia la supervivencia del modo de vida de los grupos en sus movimientos en función de las estaciones climáticas, conjugados con la organización social, en donde la relación con el ambiente está imbricada en las prácticas socioculturales. La ocupación e intervención a partir del usufructo y la posesión de determinados espacios implican una valorización sobre determinado territorio.

\section{Referencias citadas}

Adomilli, G. (2007). Terra e Mar, do viver e do trabalho na pesca maritima: tempo, espaço e ambiente com pescadores de São José do Norte - RS. (Tesis de Doctorado). Universidade Federal do Rio Grande do Sul, Porto Alegre, Brasil.

Bourdieu, P. (1958). Sociologie de l'Algérie. Paris: PUF.

Bunse, H. W. (1982). São José do Norte: aspectos linguísticosetnográficos do Antigo município. Porto Alegre: Editora Globo.

Cascaes, F. (1989). O Fantástico na Ilha de Santa Catarina. Florianópolis: Editora da UFSC.

Cordell, J. (2001). Marginalidade social e apropriação territorial marítima na Bahia. En Diegues, A. C.; Moreira, A. C. (Orgs.). Espaços e recursos naturais de uso comum. São Paulo: NUPAUB/USP.

Diegues, A. C. (1983). Pescadores, Camponeses e Trabalhadores do Mar. São Paulo: Ed. Ática.

Diegues, A. C. (Org.). (2000). Saberes tradicionais e biodiversidade no Brasil. Brasília: MMA. Recuperado de: http://livroaberto.ibict.br/bitstream/1/750/2/Biodiversidade $\% 20 \mathrm{e} \% 20$ comunidades $\% 20$ tradicionais $\% 20$ no\%20Brasil.pdf;Saberes (acceso 13.09.2019).

Editora do Brasil. (2019). Mapa Regiáo Sul: político - Projeto Jimboê. Recuperado de: http://www.editoradobrasil.com. $\mathrm{br} /$ jimboe/galeria/imagens/index.aspx?d=matematica\&a $=4 \& \mathrm{u}=2 \& \mathrm{t}=$ imagem $($ acceso 13.09 .2019$)$.

Ihering, H. V. (2003). A lagoa dos Patos no século XIX. Rio Grande: Ed. Ecosciencia.

Kern, A. (1994). Antecedentes indigenas. Porto Alegre: Editora da universidade /UFRGS.

Lima, R. K. (1999). Polícia, justiça e sociedade no Brasil: uma abordagem comparativa dois modelos de gerenciamento de conflitos no espaço público. Revista de Sociologia e Politica, 13, 23-38. 
Lima, R. K. (2004). Os cruéis modelos jurídicos de controle social. Insight-Inteligência, 6(25), 131-147.

Martins, C. A. (2002). No trabalho dos pescadores artesanais a Lagoa dos Patos vive e dá vida. Scripta Nova, 4, 1-20.

Niederle, P. A., y Grisa, C. (2006). Transformações sócioprodutivas na pesca artesanal na Lagoa dos Patos RS. Revista Eletrônica do Mestrado em Educação Ambiental, $16,85-106$.

Orlando, A. M. (1989). Alguns aspectos da pesca fazem Camarão não está localizado em Lagoa dos Patos, Rio Grande do Sul, Brasil. Rio Grande: CIRM - FURG (No publicado).

Queiroz, M. I. P. (1972). O campesinato brasileiro. Petrópolis: Ed. Vozes.

Rial, C. S. (1988) Mar de Dentro. A transformação do Espaço Social na Lagoa da Conceição. (Tesis de Maestría). Universidade Federal do Rio Grande do Sul, Porto Alegre, Brasil.
Santos, C. F., Puccinelli, V. R., Oliveira, C. L., Mascarello, M. A., y Machado, C. R. S. (2016). São José do Norte (RS/Brasil): meio ambiente como mercadoria. Geografares, 2(22), 82-94. Recuperado de: http://www. periodicos.ufes.br/geografares/article/view/11991 (acceso 13.09.2019).

Wolf, E. (1957). Comunidades corporativas cerradas de campesinos en Mesoamérica y Java Central. Southwestern Journal of Anthropology, 13(1), 1-17. Recuperado de: https://www.ciesas.edu.mx/publicaciones/clasicos/00_ CCA/Articulos_CCA/CCA_PDF/041_WOLF_Comunidades_campesinas_corporadas_20150522.pdf (acceso 13.09.2019).

Woortmann, E. (1995). Herdeiros, Parentes e Compadres. Colonos do Sul e Sitiantes fazem Nordeste. São Paulo: Hucitec Estudos Rurais.

Woortmann, E. (1997). O Trabalho da terra e a lógica simbólica da lavoura camponesa. Brasilia: Ed. UNB. 